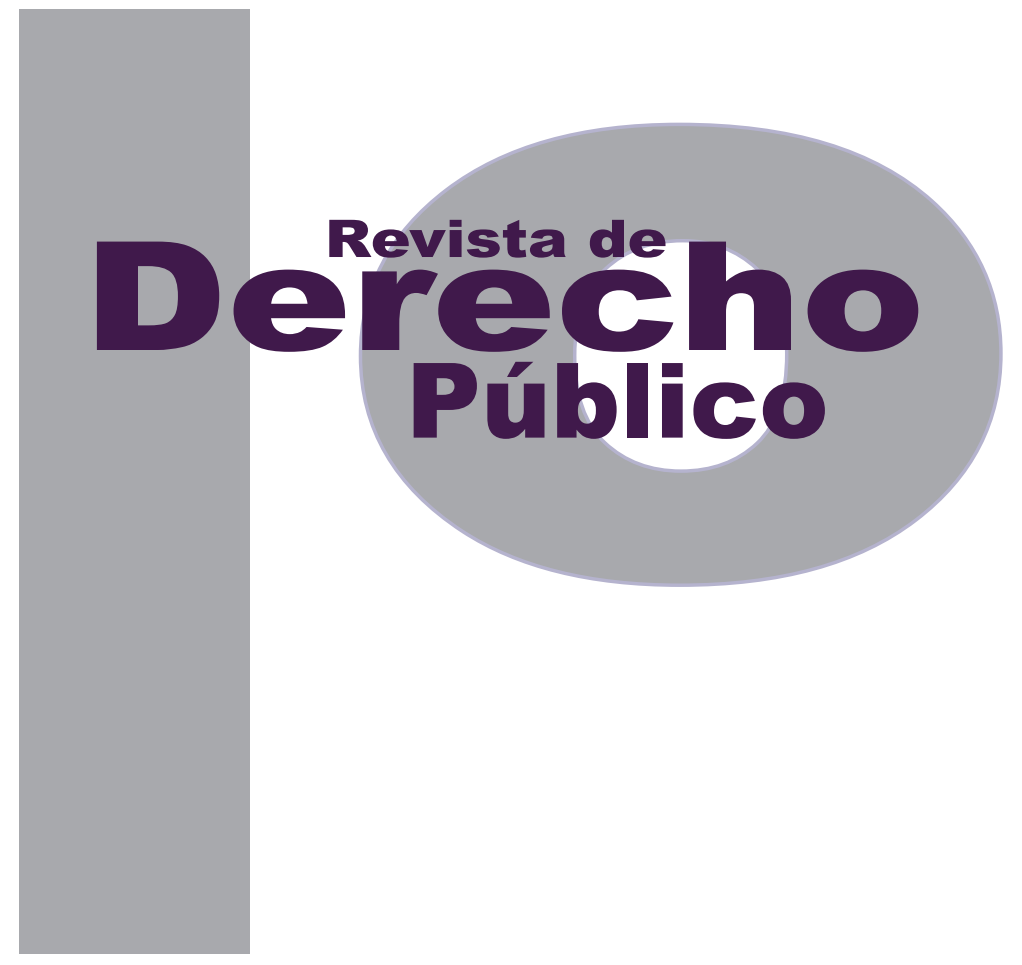

\title{
ENTRE EL (IN)DETERMINISMO “FÍSICO" Y EL "PSICOLÓGICO": ¿RESPONSABILIDAD MORAL POR ACCIONES O POR RESULTADOS?
}

\author{
ANDRÉs Felipe díaz ArANA
}

Universidad de los Andes

Facultad de Derecho

Revista de Derecho Público N. ${ }^{\circ} 30$

Enero - Junio de 2013. ISSN 1909-7778 


\title{
Entre el (in)determinismo "físico" y el "psicológico": ¿ Responsabilidad moral por acciones o por resultados?*
}

\author{
Andrés Felipe Díaz Arana**
}

\section{RESUMEN}

En la actualidad, la mayoría de las discusiones con respecto a la responsabilidad moral giran en torno al debate entre libertad y determinismo. No obstante, sucede con frecuencia que dicho debate presume, al menos, la posibilidad de atribuir responsabilidad al agente por un resultado acaecido. De hecho, rara vez suele discutirse acerca de la vigencia de los extremos de la relación sub examine, estos son: de un lado, el aspecto volitivo del agente (voluntad, dolo, etc.); de otro, el resultado acaecido en el "mundo exterior" (realidad, entorno, sociedad, etc.). Con este trabajo, también se pretende estudiar el debate entre determinismo e indeterminismo a la luz de la responsabilidad moral del agente. No obstante, el enfoque es muy preciso y estará dado en gran medida por el debate entre responsabilidad moral por acciones o por resultados. En últimas, se pretende revaluar la
Nowadays, the majority of the discussions with regards to moral responsibility are focused towards the debate between freedom and determinism. However, it is common that the same debate presupposes at least the possibility of attributing responsibility to the agent in terms of the result occurred. In fact, it is rare the discussion about the validity of the ends of the relation studied, these are: on one side, the volitional aspect of the agent (will, dolus, etc.); and on the other, the result occurred in the "exterior world" (reality, context, society, etc.). With this paper, the purpose is also to study the debate between determinism and indeterminism in the light of moral responsibility of the agent. Nevertheless, the focus is very sharp and will be given in a vast part by the debate between moral responsibility of actions or results. Basically, it is pretended to revaluate the discussion regarding determinism,

Este escrito surge a partir del trabajo final presentado en el seminario Determinismo, dado en la Universidad de los Andes durante el semestre 2012-1, a cargo del profesor Alfonso Conde.

** Estudiante de Derecho y Filosofía de la Universidad de los Andes. Monitor del área de Derecho penal, procesal penal y criminología. Miembro del grupo de estudios "Cesare Beccaría" de la Universidad de los Andes. 
discusión en torno al determinismo, de cara a la incongruencia en el juicio de imputación de resultados. Se defenderá la tesis según la cual, sea cual fuere la postura adoptada en el debate entre determinismo e indeterminismo, la pregunta por la responsabilidad moral debe estar referida a la acción, nunca al resultado.

Palabras clave: determinismo, indeterminismo, libertad, control, suerte, física, psicología, responsabilidad moral, acción, resultado. when faced to the incongruence in the judge of imputation of results. It will defend the thesis according to which, be as it may the position adopted in the debate between determinism and indeterminism, the question for moral responsibility must be referred to the action, never to the result.

KEY WORDS: determinism, indeterminism, freedom, control, luck, physics, psychology, moral responsibility, action, result. 


\section{SUMARIO}

Introducción y planteamiento del problema - I. ALGUNAS PRECISIONES PREVIAS: ¿DETERMINISMO BASADO EN LAS CIENCIAS NATURALES O EN LA PSICOLOGÍA? - A. Determinismo físico y psicológico - 1. Determinismo físico - 1.1. Popper y el argumento epistemológico - 1.2. Earman y el estudio a partir de las ciencias naturales - 1.3. Heisenberg y el principio de la incertidumbre: ¿problema físico o epistemológico? - 2. Determinismo psicológico - 2.1. La doble causalidad en Blanshard - 2.2. Robert Kane y la dualidad entre determinismo e indeterminismo en el aspecto volitivo del comportamiento humano - II. (IN)DETERMINISMO PSICOLÓGICO Y RESPONSABILIDAD POR ACCIONES: EL SIGUIENTE PASO EN LA PREGUNTA POR LA RESPONSABILIDAD MORAL - A. Indeterminismo, suerte y responsabilidad moral -

$B$. Determinismo y responsabilidad por resultados - C. Acción voluntaria y responsabilidad moral - III. CONCLUSIÓN: ¿RESPONSABILIDAD SIN RESULTADOS? - Bibliografía. 


\section{Introducción y planteamiento \\ del problema}

Al hablar de responsabilidad moral, con frecuencia surge de forma inmediata la pregunta por el determinismo. Suele suceder que, al reprochar la conducta de un agente, se refiera a la producción de un resultado que hubiera podido evitar actuando de otra forma. Inevitablemente, tendemos a identificar la responsabilidad moral con la indeterminación de los resultados posibles: el juicio de reproche a menudo se basa en la exigibilidad de actuar en contrario como forma de evitar la producción de los resultados desvalorados. La responsabilidad moral resultaría, entonces, de la producción de un resultado desaprobado, que de otra forma hubiera podido evitarse. De ahí la relevancia de la discusión en torno al determinismo, pues en un mundo en el cual los resultados fueren fijados de antemano, ¿qué sentido tendría sancionar a quien, actuare como actuare, no hubiera podido evitar el resultado acaecido? De esta forma, la discusión referente al determinismo discurre entre la posibilidad de evitar o no eventos a los que, con algún grado de arbitrariedad, llamamos resultados de nuestra conducta, acción, actuar, etc.

No obstante, pensemos por un instante en el siguiente caso:

Un niño inquieto, aprovechando un descuido de su madre, sin pensarlo dos veces, se apresura a cruzar una autopista para recoger el balón que ha caído del otro lado de la acera. Corre hacia su balón con tal suerte que un conductor muy habilidoso ha logrado esquivarlo por poco. La madre, horrorizada, se abalanza sobre el niño cuando este vuelve campante y en medio de gritos le ordena jamás volver a hacer eso. El niño, desconcertado, le pregunta a la mama qué es "eso" que ella le recrimina ${ }^{1}$.

Ciertamente, "eso" que la madre le reprocha no puede ser "salir ileso", pues de ser así la madre habría perdido la razón. ¿Cuál sería el precepto que el niño ha violado? ¿“No salgas ileso al cruzar imprudentemente una autopista"? No parece ser el resultado acaecido lo que genere un juicio de reproche en casos como este. Por el contrario, parece ser la posibilidad de un resultado adverso lo que fundamenta el reproche de la madre al niño.

Supongamos, igualmente, que el niño se queda pensando en qué fue lo que estuvo mal en su conducta: ¿su acción de cruzar imprudente o el resultado posible? Concluye que si lo reprochable es su acción, no tiene sentido que lo castiguen pues "lo hecho, hecho está"; y, si es el resultado posible, "él solo responde por sus actos". Esta ingenua forma de evadir la responsabilidad lleva al niño a preguntarse: “¿qué tal si nunca hubiera sido posible que el conductor me atropellara? ¿Qué podría reprocharme mi madre en ese caso?".

Pues bien, el razonamiento del niño ha dado con uno de los puntos más complejos en toda la historia de la filosofía de la acción y la responsabilidad moral. Una vez se enfrenta al panorama determinista, los contornos de la responsabilidad moral tienden a difuminarse y no se sabe bien cuándo tiene sentido y cuándo no. El presente

Este ejemplo no es significativamente diferente de aquel propuesto por Marcelo Sancinetti en: SANCINETTI, Marcelo. Responsabilidad por acciones o responsabilidad por resultados. Bogotá: Universidad Externado de Colombia, 1996, págs. 2-3. 
estudio pretende abordar el vasto problema preguntándose por el enfoque adecuado a la hora de hacerse preguntas como la del niño travieso: ¿qué es lo relevante en el juicio de responsabilidad?, ¿qué efectos tiene comprometerse con un determinismo ontológico para efectos de atribución de responsabilidad moral?, ¿cuál debe ser el marco de referencia para hablar de un determinismo?, ¿puede hablarse de responsabilidad por resultados?

\section{ALGUNAS PRECISIONES PREVIAS: ¿DETERMINISMO BASADO EN LAS CIENCIAS NATURALES O EN LA PSICOLOGÍA?}

Contrario a lo que usualmente sucede en la doctrina, en esta ocasión considero que sería un contrasentido definir a priori qué entiendo por "determinismo" e "indeterminismo". Esto, porque aunque la forma más clara de exponer el debate acerca de una tensión es identificando los elementos sobre los cuales versa la disputa, encuentro que gran parte de la discusión en torno al determinismo tiene sus raíces en la forma misma como entendemos tal figura. Con todo, podemos suponer - para fines metodológicos ${ }^{2}$ que determinismo implica, al menos, que todos los eventos son causados, e indeterminismo supone la postura según la cual hay, al menos, algunos eventos que no son causados. Conforme se avance en el trabajo se podrá delimitar clara-

2 A fin de cuentas, el objeto de este trabajo no es definir de una forma rígida qué pueda entenderse por "determinismo" o "indeterminismo" sino que, más bien, pretende revaluar el enfoque de la discusión misma. Esto no significa que no hayan de delimitarse los extremos del debate que lo sustenta, solo significa que no es este el objetivo primordial, sino apenas un paso necesario. mente cuál debe ser la definición de este marco de referencia empleado en la discusión sobre responsabilidad por acciones o por resultados.

\section{A. Determinismo físico y psicológico}

La disputa entre determinismo e indeterminismo puede verse desde varios enfoques. Sin embargo, en este trabajo quiero destacar dos: aquel que denomino "físico" y aquel que denomino "psicológico". El primero de ellos se centra en el estudio del mundo físico a partir de las ciencias naturales para abordar el problema, mientras que el otro está más enfocado en el estudio de la mente humana a partir de conceptos traídos por la psicología ${ }^{3}$. No es el momento de efectuar un análisis exhaustivo de los autores que pueden encajarse en cada una de las posiciones. Por el contrario, dado que esto es solo una contextualización del debate, que servirá como base para desarrollar una crítica diferente, únicamente se expondrán algunos de los autores que permiten dibujar -muy groseramente- las posturas que aquí se pretenden traer a colación. Así, dentro de aquellos autores que están en la primera alternativa, podemos encontrar a Popper, Earman y Heisenberg. Con respecto al enfoque psicológico, Blanshard y Kane ofrecen una interesante perspectiva que plantea un paralelo entre el mundo físico y el funcionamiento de la mente humana.

Aunque podría decirse que el determinismo físico frecuentemente conlleva a que se asuman

3 Entiendo psicología en su sentido más amplio: "Ciencia que estudia los procesos mentales en personas y en animales". Real Academia Española. Diccionario de la lengua española. Vigésima segunda edición 
dos posturas distintas (o bien, todos los eventos son causados; o bien, hay algún evento que no es causado y que permite hablar de indeterminismo ${ }^{4}$ ), considero que limitar de esta forma el trabajo podría resultar inadecuado porque, así planteada la disputa, la respuesta solo podría darse en términos dicotómicos: el triunfo del determinismo anularía la vigencia del indeterminismo, y viceversa. De hecho, otras posturas entienden que no necesariamente porque se demuestre la existencia de un evento que no está determinado, o de uno que sigue leyes causales rígidas, la tesis que se pretende oponer pierde vigencia. En efecto, el argumento central de quienes hacen un paralelo entre el determinismo físico y el funcionamiento de la mente es que en el comportamiento humano existen distintos ámbitos, unos determinados por las leyes físicas y otros en los cuales cabe hablar de libre albedrí $^{5}$. Tal es el claro caso de Kaney, como se argumentará, también de Blanshard, quienes sostienen que la discusión debe darse en dos niveles, en los cuales podría hablarse de distintos tipos de causalidades, y por lo tanto podrían tener vigencia ambas teorías al tiempo ${ }^{6}$.

4 Es absolutamente imprescindible aclarar lo siguiente: no se trata de que el indeterminismo por ser la negación del determinismo implique que todos los eventos son indeterminados. En términos lógicos, la negación de un universal es un existencial, por lo que los indeterministas como forma de oponerse al determinismo deben demostrar que existe al menos un evento que no está determinado (sea cual sea su definición).

5 Es colosalmente más compleja la diferencia entre ambas figuras, pero dado el escaso espacio y los limitados fines de este trabajo, bastará así planteada. Para su mayor desarrollo ver: FrankFURT, Harry. La importancia de lo que nos preocupa. En: La importancia de lo que nos preocupa. Trad. Verónica Inés Weinstabl y Servanda María de Hagen. Buenos Aires: Katz Editores, 2006, pág. 119 y ss.

6 Como se argumentará más adelante, el hecho de que Blanshard afirme que en ambos casos hay determinismo, no implica que de demostrarse que en el plano físico haya determinismo se derive lo mismo para el plano psicológico. Por esta razón, se considera que tal autor
Esto es de suma importancia para la pregunta sobre la responsabilidad moral, pues de entrada debe entenderse que la pugna entre el (in) determinismo físico y psicológico es tan compleja que, aunque el determinismo físico pueda oponerse al indeterminismo psicológico, el indeterminismo psicológico no se opone al determinismo físico (sino que, muy por el contrario, en muchas posturas resulta un presupuesto). Luego, antes de abordar la posibilidad de imputar responsabilidad por los resultados, es menester que se delimite claramente cada postura aquí expuesta.

\section{Determinismo físico}

\subsection{Popper y el argumento epistemológico}

Karl Popper, quien se opuso a la visión de físicos como Bohr, y en general a la visión imperante sobre la mecánica cuántica, va a ser el punto de partida para desdibujar el trasfondo de este trabajo porque desplaza por completo la discusión con respecto al determinismo del plano físico al epistemológico. En efecto, Popper explica que una cosa es el determinismo objetivo -es decir, del objeto mismo- y otra el determinismo accesible al sujeto del conocimiento, es decir, si le es posible al sujeto, a partir del conocimiento actual, predecir cursos causales futuros ${ }^{7}$. Con esta división, este autor pretende hacer valer una postura según la cual el problema central

ofrece una sólida base que es común a los enfoques basados en la psicología, independientemente de su conclusión final.

7 POPPER, Karl. Conocimiento: subjetivo contra objetivo. En: MilleR, David (comp.). POPPER: Escritos selectos. México: Fondo de Cultura Económica, 1995, pág. 63 y ss. 
no está en la realidad o no del determinismo físico, sino que el problema epistemológico verdadero radica en la accesibilidad de tal determinismo al sujeto que conoce.

Esto lleva a Popper a argumentar que no es útil ni susceptible de argumentación racional sostener la tesis según la cual todo lo que sucede en el mundo resulta inevitablemente de condiciones precedentes, pues sostiene que el debate en torno al determinismo físico no tiene sentido más allá de su (im)posibilidad de verdadero conocimiento por parte del sujeto. La paradoja que lleva a Popper a argumentar en contra del determinismo desde un plano epistemológico creo que podría resumirse de la siguiente manera. El método de la predicción solamente es viable para aquellos casos en los cuales la predicción misma no afecta la ocurrencia futura de Ios eventos; en el caso del determinismo físico, el sujeto que hace la predicción es él mismo objeto de estudio. Por esto, si fuéramos capaces de saber con plena certeza el ocurrir futuro, la conciencia no sería inmune a tal conocimiento, afectando inevitablemente la ocurrencia de eventos futuros y por ende haciendo imposible la predicción objetiva tal y como pretende ser defendida ${ }^{8}$. Popper afirmaría que el determinismo es un problema esencialmente epistemológico, por lo tanto podríamos caracterizar su postura como indeterminismo epistemológico.

8 PoPper, Karl. The Open Universe. Capitulo I "Kinds of Determinism". Londres: Routledge, 1991, pág. 15 y ss.

\subsection{Earman y el estudio a partir de las ciencias naturales}

Contrario al determinismo como problema epistemológico, Earman enfoca su estudio en la discusión sobre determinismo desde un plano físico, basándose en gran medida en las ciencias naturales y las leyes físicas. La postura del determinismo físico, defendido por ciencias naturales puede verse así: existen leyes naturales, generales y sin excepciones, que hacen que las cosas ocurran de cierta manera, explican por qué ocurrieron así y permiten predecir cómo ocurrirán bajo las mismas circunstancias en un futuro ${ }^{9}$. La idea "Dios no juega a los dados", Ilevada a sus extremos, conlleva el pensamiento según el cual las leyes físicas, que gobiernan los fenómenos físicos, son la causa de todo lo que sucede en el plano físico. Y, como nosotros tenemos (al menos) una dimensión física, estamos sometidos inevitablemente a esas leyes causales.

Earman sigue una corriente según la cual la respuesta a la disputa entre determinismo y libre albedrío está dada en el plano físico, a partir del estudio de las ciencias naturales. Tal corriente, cuyas raíces están en Hume, Mill y también contemporáneos como David Lewis $^{10}$, considera que las leyes naturales son el mejor sistema de regularidades que permiten sistematizar todos los eventos en la historia universal. Independientemente de si las leyes son una derivación onto-

Earman, John. A Primer on Determinism. Capítulo II "Defining Determinism". Dordrecht: Reidel Publishing Company, 1986, pág. 9 y ss.

10 LEWIS, David K. Humean Supervenience debugged. En: LewIS, David. Papers in Metaphysics and Epistemology. Nueva York: Cambridge UP, 1999, pág. 224 y ss. 
lógica, de tal forma que es la realidad la que las dicta y no al revés, o de si la relatividad general permite hablar de singularidades que no están regidas por leyes universales (argumentos que el propio autor expone a favor del libre albedrío), Earman -contrario a Popper- considera que el plano de discusión puede y debe ser físico a partir de las ciencias naturales.

\subsection{Heisenberg y el principio de la incertidumbre: ¿problema físico o epistemológico?}

En igual sentido va la propuesta de Heisenberg quien, a partir de la mecánica cuántica, aborda el problema del determinismo desde el plano de las ciencias naturales, en particular de la física. El problema del que se ocupa Heisenberg radica en el principio de la incertidumbre como defensa al indeterminismo ${ }^{11}$. De entrada, puede decirse que el estudio a partir de las ciencias naturales sobre el problema del determinismo hace asemejarlo a Earman (en su enfoque, no en su respuesta); no obstante, el lugar al que llega con su teoría sobre el límite a la hora de medir la posición y el momento de una partícula en un mismo instante del tiempo hace pensar que tiene un fuerte arraigo en los problemas epistemológicos traídos a colación por Popper.

No creo que Heisenberg sea un indeterminista epistemológico ni uno totalmente físico. Aun

11 Para la formulación del principio, así como sus consecuencias, ver: Heisenberg, Werner. Physics and Philosophy. Capítulo II "The History of Quantum Theory". Nueva York: Harper and Brothers Publishers, 1958, pág. 30 y ss; Heisenberg, Werner. Physics and Philosophy. Capítulo III "The Copenhagen Interpretation of Quantum Theory". Nueva York: Harper and Brothers Publishers, 1958, pág. 44 y ss. cuando se pueda pensar que por plantear la imposibilidad de medir el momento y la posición actual de una partícula al mismo tiempo en que se pretende medir su posición futura, Heisenberg se adscriba al problema del determinismo desde un enfoque epistemológico, creo que su argumento va más encaminado al estudio de las leyes físicas que rigen el mundo de forma inmutable y universal. Por estas razones, aunque este autor mantiene un claro enfoque basado en las ciencias naturales, no parece claro que deba ser adscrito a ninguno de los dos bandos anteriormente expuestos.

\section{Determinismo psicológico}

Distintas visiones pueden ejemplificar el dualismo entre física y psicología como forma de abordar el problema del determinismo a la hora de preguntarse por la responsabilidad moral. Sin embargo, en mi opinión, es más interesante la disputa en torno a aquellos autores que, como Kane y Blanshard, sostienen que aunque posturas como las de Earman y Heisenberg puedan ser válidas en el plano físico, existe un plano psicológico que no puede ser olvidado a la hora de hablar de responsabilidad moral ${ }^{12}$. En este momento abordaré la figura del determinismo psicológico, que sostiene que determinismo físico y libre albedrío como propiedad volitiva del hombre pueden ser ambos verdaderos. Cada autor va a proponer una teoría distinta a favor

12 Para mayor profundidad, ver: MoyA, Carlos J. Alternativas, responsabilidad y respuesta a razones. En: Ideas y Valores. Universidad Nacional de Colombia, vol. 58, no. 141, 2009. Disponible en: http://www. revistas.unal.edu.co/index.php/idval/article/viewFile/12768/13370. Consultado por última vez el día 21 de marzo de 2012. 
o en contra del determinismo, pero los une el que ninguno ubica su estudio únicamente en el plano físico. Por el contrario, aceptan que -aunque en algún grado estamos determinados causalmente por la física- el libre albedrío puede estudiarse en un nivel distinto (en particular, en las voliciones humanas). Lo más importante $-\mathrm{y}$ que habrá de ser destacado en esta parte- no es el hecho de que ambos sean considerados deterministas o indeterministas (lo cual ya da para un ensayo aparte) sino el gran aporte que conlleva desplazar el estudio del problema desprendiéndolo del plano físico hasta ubicarlo en el plano psicológico. Este es el primer gran paso si se quiere abordar adecuadamente la pregunta por responsabilidad moral, tanto de la acción como del resultado.

\subsection{La doble causalidad en Blanshard}

Independientemente de que pueda dudarse de que Blanshard fuera en realidad un determinista o un indeterminista, creo que él debe ser el punto de partida para superar el arraigo a las ciencias naturales principalmente porque, a fin de cuentas, toda su lucha se basa en un argumento que busca superar la física como forma única de abordar el problema. Como se verá, la dualidad de cursos causales con la cual pretende separar los descubrimientos aplicables al plano físico de las consecuencias extrapolables a un plano psicológico, supone un gran paso a la hora de delimitar el marco de estudio del problema con miras al comportamiento humano.

En efecto, aunque al abordar el problema de los cursos causales físicos Blanshard aporta múltiples argumentos en defensa del determinis- $\mathrm{mo}^{13}$, lo verdaderamente importante para este trabajo es su tesis de una radical separación entre procesos físicos y procesos mentales ${ }^{14}$. Esto es importante porque destaca un aspecto fundamental: un estudio basado en la mente del sujeto activo no omite las consideraciones que aportan las ciencias naturales; por el contrario, las integra, y estudia si todas ellas tienen las mismas repercusiones en la psicología del comportamiento humano. Con este argumento, el autor pretende establecer una dualidad de planos en los cuales se ve inmersa la discusión sobre determinismo, siendo este el paso necesario para hablar de responsabilidad moral dentro de un contexto de determinismo físico.

Cuando Blanshard explica que el ser humano tiene dos facetas - una física y una mental-que pueden y deben ser tratadas distintamente, lo hace con un claro propósito: argumentar que, a pesar de que pueda considerarse que en el plano físico estemos condicionados por leyes físicas (al estilo del estudio realizado por Earman), de todos modos los procesos mentales se encuentran en un plano distinto. Independientemente de que defienda igualmente el determinismo en ambos niveles ${ }^{15}$, este argumento de dualidad entre el plano físico y el psicológico puede ser un gran fundamento para otros autores que ahondan más en los procesos pura-

13 Siendo uno de los más importantes la diferencia entre el todo y la parte, empleado para superar el argumento del indeterminismo cuántico. Ver: Blanshard, Brand. The Case of Determinism. En: Determinism and Freedom in the Age of Modern Science. Hook, S. (ed.). CollierMacmillan: Nueva York, 1958, pág. 26.

14 Ibid., pág. 28

15 Ver sus conclusions en: BLANSHARD, Brand. The Case of Determinism. En: Determinism and Freedom in the Age of Modern Science. Hook, S. (ed.). Collier-Macmillan: Nueva York, 1958, pág. 30 y ss. 
mente volitivos del ser humano (como Kane, por ejemplo).

Es así como, dentro del mapeo de las distintas posturas sobre determinismo e indeterminismo, parece que el determinismo de Blanshard -tomando en cuenta las consideraciones aquí traídas a colación- representa un primer paso hacia la fundamentación de la importancia de la psicología como aspecto innegable del comportamiento humano. Con ello, la pregunta por la responsabilidad moral no solo vuelve a tener importancia, sino que recobra el sentido mismo que había perdido en un plano de discusión puramente físico. Al fin y al cabo, fuere determinista o indeterminista el enfoque físico adoptado, lo cierto es que la pregunta por responsabilidad moral solo tiene sentido una vez se asume que: independientemente de que estemos regidos en tanto seres físicos por leyes físicas o no, en el nivel psicológico una distinta relación aplica entre la volición y el acto. El desprendimiento de la pura ontología natural no solo es un gran avance hacia la pregunta por la responsabilidad moral, sino que en realidad representa el único panorama en el cual tal pregunta puede tener sentido alguno.

\subsection{Robert Kane y la dualidad entre determinismo e indeterminismo en el aspecto volitivo del comportamiento humano}

Es en gran medida la dualidad propuesta por Blanshard la que da cierto sentido a la teoría de Robert Kane, quien desde una perspectiva plenamente psicológica afirma que, a pesar de que muchos eventos de nuestro diario vivir es- tén causados, el libre albedrío (y el indeterminismo) se presenta en algunos eventos (mentales) muy importantes ${ }^{16}$. Parece ser que el problema al que Kane apunta en ningún momento es la atribución de responsabilidad por resultados, sino algo que es muy anterior: atribución de responsabilidad sobre la misma elección. En este sentido, el escrito de Kane es un ensayo sobre la psicología humana, al punto que usa conceptos traídos de la neurociencia (como saltos en redes neuronales y procesos de sinapsis ${ }^{17}$ ) para fundamentar su posición.

Con posturas como esta, la pregunta por el determinismo cambia de enfoque. Ya la disputa no es si la física puede o no ser un argumento en contra del libre albedrío, no se trata de si en un plano físico, o incluso epistemológico (por no mencionar el metafísico), somos libres o no. Se trata de reconocer el plano dual que tiene el ser humano, entre lo físico y lo psíquico, de llevar la doble causalidad de Blanshard a pleno desarrollo.

Robert Kane explica que aun cuando en los eventos cotidianos estemos causalmente determinados, existe un espacio para el libre albedrío en el plano de las elecciones y la toma de decisiones del ser humano. Con posturas como la de Kane, y sus acciones de autoformación, la pugna por el determinismo cambia de foco radicalmente, dándole cabida a preguntas por la responsabilidad moral y la imputación de accio-

16 KANE, Robert. Responsibility, Luck and Chance. En: Journal of Philosophy, vol. 96, No. 5, 1999, pág. 220 y ss.

17 Ver: Kane. Op. cit., pág. 225. 
nes que no están subordinadas al avance de las ciencias naturales (al menos no directamente).

\section{II. (IN)DETERMINISMO PSICOLÓGICO Y RESPONSABILIDAD POR ACCIONES: EL SIGUIENTE PASO EN LA PREGUNTA POR LA RESPONSABILIDAD MORAL}

A pesar de que Kane pueda abogar por un indeterminismo y Blanshard por un determinismo, mientras que Popper habla de un indeterminismo epistemológico y Earman y Heisenberg basan su estudio en las ciencias naturales, lo importante no son las diferencias en la respuesta, sino en el camino para llegar a tales respuestas, partiendo de la formulación misma de la pregunta. En la formulación de la pregunta por el determinismo se encuentran las bases para la respuesta a la interrogación por la responsabilidad moral de acciones o resultados. Habiendo expuesto dos polos del debate en torno al determinismo, en adelante habrá de tomarse como trasfondo de los argumentos a favor de la responsabilidad por acciones el determinismo psicológico. Esto, no solo porque es el único que permite abordar el particular de una forma coherente, sino porque a través del estudio de la relación entre volición y acción se podrá llegar a la conclusión según la cual solamente podemos ser tenidos como responsables de nuestros actos, no de los efectos que nuestros actos causen en el mundo.

Según Hegel, solo puede llamarse imputación -en estricto sentido- a aquello que en una conducta puede ser reconocido como mío ${ }^{18}$. Es

18 Ver: Hegel, Georg W. Líneas fundamentales de la filosofía del Derecho. Trad. F. E. G. Vicent. Madrid, Revista de Occidente, 1935. momento de estudiar si los resultados -en estricto sentido- pueden ser imputados al agente, o si por el contrario, solo la acción es verdaderamente suya. En lo que sigue pretendo estudiar a profundidad lo que puede ser llamado 'primer dogma de la causalidad': para que pueda hablarse de responsabilidad debe, por lo menos, poder constatarse que la acción causó el resultado. Es decir, tomando como base un enfoque psicológico en el debate entre determinismo e indeterminismo, se pretende ahora estudiar si aún a pesar de mantenerse un indeterminismo psicológico al estilo de Kane, puede sostenerse que somos responsables de algo más que de nuestras propias acciones.

\section{A. Indeterminismo, suerte y responsabilidad moral}

Kane ha expuesto en su texto Responsability, Luck and Chance, una variedad de ejemplos en los que se cuestiona tácita y expresamente el papel de la suerte en la atribución de responsabilidad al agente. Su argumento parte de la constatación de una paradoja en la forma como el determinismo ha sido abordado frecuentemente. Para ello propone el siguiente ejemplo: Jane se debate entre ir a Hawaii o Colorado para sus vacaciones; luego de mucho pensarlo, gradualmente se inclina a favor de Hawaii ${ }^{19}$. Si se estudia tal decisión bajo el foco determinista, no había otra opción real pues Colorado nunca fue posible dado que Jane se encontraba determinada a priori para escoger Hawaii. Así las cosas, no parece haber ningún tipo de responsabilidad sobre la decisión de Jane. Sin embargo, el

19 Parafraseado de: KANE, Robert. Op. cit. pág. 220-221. 
enfoque indeterminista es igualmente insatisfactorio: suponiendo que Jane estuviere absolutamente indeterminada a la hora de elegir su lugar de vacaciones, su decisión entre Colorado y Hawaii no pudo deberse a otra cosa más que a la suerte ${ }^{20}$.

Dejando a un lado las consideraciones de Kane sobre los procesos mentales que llevaron a Jane a elegir una opción, debe destacarse que este punto tiene que ver con la forma como entendamos responsabilidad de acciones y de resultados. Esto es de suma importancia pues al tratar las acciones de autoformación, Kane equipara el ejemplo del esposo con el de la mujer trabajadora $^{21}$, en los cuales no se debate lo mismo. En el caso del esposo, la pregunta es si el quiebre de la mesa (resultado) es atribuible a este; en el caso de la mujer trabajadora, la pregunta es si el abandono de la víctima (acción) es atribuible a la esta ${ }^{22}$. Aunque Kane los diferencia en ocasiones, termina tratándolos de manera similar toda vez que ambos tuvieron éxito en sus pretensiones. No obstante, ¿es suficiente hablar de querer y éxito como fórmula de atribución de responsabilidad?

En los ejemplos que yo entiendo como atribuciones de responsabilidad por resultados, Kane explica que "actuar de otra manera (no matar al

20 O, en términos del propio Kane, "saltos neuronales aleatorios". KANE, Robert. Op. cit., pág. 223.

21 Tales ejemplos aparecen en el acápite VI de KANE, Robert. Op. cit., pág. 233 y ss.

22 Para que fueran equiparables, debería preguntarse si el hecho de que maten a la víctima (resultado) es atribuible a la acción de la mujer al abandonarla. Pero en este caso se pregunta si la decisión misma de irse es atribuible a la mujer. ministro, no romper el vidrio y no resolver el problema) es un fallo en la acción debido al azar, la suerte o la casualidad y no una decisión del agente" ${ }^{23}$. No obstante, creo que el punto central parte de una diferencia importante: en los ejemplos de Kane, no matar al ministro no supone un problema en la acción sino en el resultado $^{24}$. ¿Es posible decir que estos dos agentes se hagan "responsables" de su voluntad y de su intención de hacer lo que se propusieron pero que no son "responsables" de los resultados obtenidos? Yo creo que sí.

Sin embargo, se quiere destacar algo que Kane omitió considerar en el texto referenciado: la "suerte en la forma en que las acciones resultan" 25 no puede tener relevancia alguna en la atribución de responsabilidad. Esto se debe, en el fondo, a que la responsabilidad no se predica de los resultados, que por su naturaleza son ajenos a nuestro control, sino de aquello que podemos controlar, a saber: las acciones.

Así pues, tradicionalmente han sido dos los argumentos que han sostenido el rechazo a la suerte como factor relevante a la hora de determinar la responsabilidad moral ${ }^{26}$. El primero trae a colación la relación de dependencia entre lo que podemos controlar y aquello de lo cual podemos ser tenidos por responsables. El segundo, un desarrollo del primero, supone que lo único

\footnotetext{
23 KANE, Robert. Op. cit., pág. 235 y ss.

24 Kane, Robert. Op. cit., pág. 334 y ss.

25 Expresión de NAgel, Thomas. Moral Luck. En: NAgel, Thomas. Mortal Questions. Nueva York: Cambridge UP, 1993.

26 Así para: FERRANTE, Marcelo. Recasting the Problem of Resultant Luck. En: Legal Theory, vol. 15, 2009, pág. 267.
} 
que en estricto sentido podemos "controlar" son nuestras propias acciones. Lo que de nuestras acciones se deriva en el mundo no puede ser controlado por nosotros sino mediante la evitación de la acción misma. Bajo este punto de vista, si a un hombre solo se le puede tener por responsable de aquello que controla, entonces únicamente puede predicarse responsabilidad por la propia acción. El punto que suele ser sensible está dado por la pregunta sobre si debe responder más aquel que logra su objetivo, que aquel que -realizando la misma acción- falla en su objetivo. Con un ejemplo:

En el mundo M1, Andrés quiere matar a su enemigo Carlos. En el mundo M2 ${ }^{27}$, Bernardo quiere matar a su enemigo Carlos. En ambos mundos Andrés y Bernardo disparan contra Carlos. En M1 la bala de Andrés impacta el cráneo de Carlos produciendo su muerte instantánea. En M2 la bala de Bernardo impacta contra una paloma que atravesaba la trayectoria entre el cráneo de Carlos y la pistola al momento en que esta fue accionada, saliendo Carlos ileso. Los mundos M1 y M2 solo se diferencian por la circunstancia descrita.

La pregunta concreta es, pues, la siguiente: ¿merece mayor atribución de responsabilidad moral Andrés que Bernardo? Para resolverla se deberá tener en cuenta qué debe primar: la capacidad de control, o el daño acaecido sobre la víctima. De un lado se esgrimen argumentos como "no es posible que algo fuera del control de Andrés y de Bernardo suponga una variación en el juicio de responsabilidad, toda vez que el fundamento de la responsabilidad es la capa-

27 Me valgo de la dualidad de mundos al estilo de Mele, citado en: KANE, Robert. Op. cit., pág. 221 y ss. cidad de actuar en contrario". Del otro, es frecuente escuchar argumentos como "no es lo mismo intentar matar, que en efecto matar".

Adoptando un enfoque de "semideterminismo" psicológico, similar a Kane, creo que el punto no se va a resolver mediante una remisión a la física. Parece evidente que -a menos que la paloma volara más rápido que la velocidad de la luz- nuestro caso en nada atañe a las leyes naturales (pues se aplican sin diferencia en ambos casos). No parece adecuado equiparar el comportamiento humano a aquel apreciable en los demás seres de la naturaleza, pues a diferencia de aquellos este transcurre en dos niveles. Luego, la pregunta por el determinismo en el nivel básico de la experiencia física no tiene relevancia alguna, pues sea o no cierto, lo importante es lo que ocurre "dentro de la mente". En términos más precisos, lo principal para resolver la presente inquietud radica en la relación entre volición y acción, no entre acción y ley natural. El punto no trata sobre la posibilidad de que una vez disparada la bala, pueda o no predecirse el resultado futuro a partir de las leyes naturales y la física moderna.

Por el contrario, creo que lo relevante consiste en determinar la responsabilidad de forma apriorística, esto es, eliminando el papel de la suerte en el acaecimiento del resultado, lo que supone determinar la responsabilidad sin tener en cuenta el resultado mismo. Un "semideterminismo" como el de Kane parece ofrecer la posibilidad para preguntarse por la responsabilidad moral de las acciones, sin que consideraciones en torno al determinismo frente al resultado resulten contradictorias. 
Parecen haber dos tipos de relaciones en el ejemplo propuesto: la primera tiene que ver con el evento 1 "disparo" y el evento 2 "muerte de Carlos". Si atendemos a tal relación únicamente, el determinismo físico puede darnos una respuesta valiosa: cada vez que se dispare bajo tales o cuales condiciones y la bala impacte el cráneo de un ser humano, producirá su muerte explicable y predecible por las leyes “x”, “y”, “z”, etc. Sin embargo, en cuanto a la posibilidad de evitar el resultado una vez se ha producido el disparo, Andrés y Bernardo no tienen responsabilidad alguna pues el determinismo físico ha descartado toda relevancia de su actuar en tal relación.

Empero, no es esta la relación relevante a la hora de atribuir responsabilidad moral. Por el contrario, la pregunta por la responsabilidad tiene que ver con aquella relación entre Andrés y Bernardo, de un lado, y el disparo, de otro. Lo importante no es la ley física que explica la relación entre la bala y la muerte de Carlos, sino aquella relación que hay entre la voluntad de Andrés y Bernardo de matar a Carlos, y el evento de la muerte de Carlos. Tal relación no puede sino versar sobre un mismo y único punto, a saber: la acción ejecutada por Andrés y Bernardo.

\section{B. Determinismo y responsabilidad por resultados}

Aún si se acepta que la suerte no puede jugar un papel en la atribución de responsabilidad, al ser por definición una circunstancia fuera de nuestro control, debe igualmente preguntarse por el grado de control -de forma general- sobre los resultados. Es decir, ¿independientemente de que la paloma haya intervenido o no, Andrés - Bernardo controlaron en algún momento el resultado "muerte de Carlos"? Pongamos otro ejemplo:

Supongamos que el profesor Alfonso le pide al estudiante Benito que encienda la luz del aula de clases con el fin de dar inicio a la sesión. Benito, muy juiciosamente, se levanta de su silla en dirección al interruptor, y al alcanzarlo lo activa pero nada sucede.

La pregunta relevante en este caso es: ¿puede el profesor Alfonso reprocharle a Benito el incumplimiento de su orden? Por supuesto, para responderlo es necesario preguntar: ¿ha hecho Benito lo que el profesor Alfonso pidió? Si la respuesta es "Benito no ha cumplido la orden del profesor Alfonso porque la luz no ha sido encendida", Benito merece ser castigado. Si, por el contrario -y como creo-, la respuesta es "la orden del profesor Alfonso, literalmente entendida, no tiene sentido", se entenderá el porqué de la respuesta más natural: Benito no merece sanción alguna.

Asumiendo que la luz no fue encendida tras la acción de Benito (mover el interruptor), supongamos que el defecto en la consecución del resultado esperado se debe a que el electricista encargado de reemplazar los fusibles cada bimestre ha olvidado cumplir su tarea y esto ha producido que el fusible en cuestión se haya quemado. Cabe hacerse dos preguntas de suma importancia. La primera y menos problemática es: ¿a quién es atribuible el defecto en la producción del resultado? En este punto creo que no habría mayor debate en aceptar que el electricista debe responder por la ausencia del re- 
sultado esperado, pues Benito ha hecho todo lo posible por asegurarlo. La segunda, mucho más polémica es: ¿hubiera habido diferencia alguna en el juicio de atribución de responsabilidad si Benito nunca se hubiera levantado de su silla?

Esto nos lleva al punto en cuestión: en un mundo en el cual se asumiera un determinismo a partir del resultado, Benito no debería responder de forma alguna aun cuando hubiera hecho caso omiso a la orden del profesor. Es decir, asumiendo que jamás se iba a producir el resultado esperado: ¿qué sentido tiene reprochar a Benito el hecho de que nunca hubiera efectuado ninguna acción para asegurarlo? Esto es, en el fondo, el punto que ha motivado el debate entre determinismo y responsabilidad moral durante gran parte de su historia.

Lo cierto es que cuando se explica que la orden emitida por Alfonso a Benito -literalmente entendida - no tiene sentido alguno, se apunta a un argumento muy simple: si Alfonso exigiera a Benito que asegurara la producción del resultado esperado -luz encendida- Benito solo podría responder que eso no le compete, pues lo único que puede hacer es mover el interruptor. Sin embargo, esto es superfluo y parece ser evidente que cuando Alfonso emitió la orden "Benito, enciende la luz", en realidad se refería a "Benito, mueve el interruptor de la luz". Alfonso debería estar al tanto de que su orden, aún a pesar de las apariencias, suponía una obligación de medio -mover el interruptor-y no de resultado - que la luz sea encendida-.

Tampoco podría ser distinto el caso en el cual Benito fuera electricista. En ese escenario, tam- poco podría asegurar la producción del resultado, pues podría cambiar el fusible y aún así no prenderse el bombillo debido a una falla en el suministro de energía. Así, podemos pensar en una regresión al infinito en la cual Benito fuera desde empleado de la fábrica de suministro de energía hasta minero, y sin embargo nunca podría cumplir con la simple orden de encender la luz. A pesar de esto, no parece coherente que Benito sea sancionado porque la voluntad de su profesor no se concretó en el resultado esperado.

En últimas, esto implica que con la orden -interpretada bajo los parámetros aquí propuestosAlfonso solo pretende que Benito haga lo que esté a su alcance para que la luz sea encendida. Se entiende entonces por qué, a pesar de que la luz nunca hubiera sido encendida, el profesor Alfonso se hubiera enfadado ante la indiferencia de Benito frente a su orden. La objeción obvia es: una vez Benito explique al profesor que no atendió la orden dada porque la luz nunca se hubiera encendido, el profesor probablemente hubiera cesado en su reproche. Por supuesto, siempre cabe la posibilidad de que el profesor Alfonso se enfadara por el incumplimiento de su orden, pues a Benito se le ordenó -debidamente interpretado- que se desplazara hasta el interruptor y lo activara, independientemente de que la luz se encendiera o no. Este reproche, propio del incumplimiento de la orden que legítimamente fue emitida por un superior, es otra evidencia que soporta el hecho de que la responsabilidad de Benito es de medio y no de resultado: el resultado de la acción ni es exigible ni, por lo tanto, su no producción sirve como excusa para no ejecutar la acción mandada. 
Lo cierto es que el hecho mismo de que sea requerida una explicación para justificar la ausencia de acción implica que la responsabilidad gira en torno a la acción y no en torno al resultado. De lo contrario, tendríamos que aceptar como razonable que el profesor persistiera en su reproche por la sola razón de que el resultado esperado no ha sido el obtenido. El pobre Benito no tendría más opción que resignarse ante el triste panorama de no poder controlar la producción de resultados... nunca.

\section{Acción voluntaria y responsabilidad moral}

Hasta el momento parece seguro concluir que la buena o mala suerte no puede ser tomada como causal de variación en el juicio de responsabilidad. Además, habiendo analizado la estructura de las órdenes con sentido de cara a la posibilidad de aseguración de resultados, podemos concluir también que los resultados nunca son del todo controlables. Si esto es así, la expectativa de aseguramiento de un resultado tampoco puede ser el eje del juicio de responsabilidad moral. Como es apenas evidente, tales conclusiones están soportadas -en el fondo-por un solo argumento: para que pueda existir un juicio de responsabilidad coherente, el agente debe contar con un control suficiente sobre el objeto del juicio de responsabilidad, bien sea para asegurar su producción o para evitarla. Esto, no es otra cosa que afirmar que para que pueda existir un reproche sobre la conducta del agente, esta debe encontrarse en cierta relación con su voluntad. De tal forma que en todo juicio de responsabilidad subyacen dos reproches: el pri- mero, es que obró con control de la situación de tal forma que pudo haber actuado en contrario; el segundo, corolario del primero, es que si pudo haber actuado en contrario, y obró de la forma reprochada, obró voluntariamente.

De aquí es fácil dejarse llevar y afirmar que la responsabilidad tiene como eje la producción de un resultado voluntario. Así lo hacen, tanto Kane como Blanshard cuando cada uno en su forma particular, llevan el determinismo psicológico a sus extremos al afirmar que aun cuando el resultado pueda estar asegurado de antemano, la responsabilidad moral subsiste porque el agente tuvo éxito en sus pretensiones ${ }^{28}$. De cualquier forma, esto sería rebatible si se constatara - como en efecto se constata- que existen casos en los cuales la producción de resultados involuntarios genera responsabilidad.

No obstante, decir que hay casos en los cuales la producción de resultados involuntarios genera responsabilidad, es impreciso. Supongamos que Alberto, conduciendo hacia su casa, involuntariamente atropella a Boris y le causa la muerte. ¿Debe tenerse como responsable a Alberto por la muerte de Boris?

La respuesta más sensata es: hacen falta datos. Pero datos sobre el resultado como tal no hacen falta, Boris está muerto y en tanto resultado hasta ahí puede ser relevante. Sin embargo, hacen falta datos sobre la forma en que sucedieron los hechos - diría el investigador prudente-. Estos datos no son más que la constatación de que Alberto iba con exceso de velocidad, borra-

28 KANE, Robert. Op. cit., págs. 238-240; BLANSHARD, Op. cit., págs. 27-30. 
cho y no contaba con licencia, por ejemplo, para que el juicio de responsabilidad sea siquiera posible. Si Alberto manejaba prudentemente, y el peatón suicida se lanzó sobre su carro, no parece ser adecuado atribuir responsabilidad al primero por la muerte del segundo. Pero el resultado fue el mismo, y en ambos casos sería igualmente involuntario. Sin embargo, parece ser claro que no es el resultado involuntario per se el que media a favor o en contra en el juicio de responsabilidad, sino que lo relevante es la acción de uno u otro agente. Sigue siendo la misma acción la que, unas veces prudente otras imprudente, produce un resultado voluntario o involuntario, sin que sea el resultado disvalioso (muerte del peatón) el que justifique la sanción en los casos de "resultados involuntarios"29.

Nuevamente, encontramos en casos como estos -en los cuales a primera vista lo único reprochable parece ser el acaecimiento del resultado- un sólido fundamento para asegurar que lo relevante en el juicio de responsabilidad es la acción.

\section{CONCLUSIÓN: ¿RESPONSABILIDAD SIN RESULTADOS?}

Aunque es cierto que va en contra de la opinión corriente, y por ello es difícil de aceptar, no constato razón alguna para que alguien deba responder más por el solo hecho de que ha obtenido

29 Sobre si la voluntad en el actuar juega un papel en estos casos de resultados involuntarios, no puede decirse nada más que lo siguiente: se considera que el actuar imprudente fue en cierto sentido voluntario porque voluntariamente decidió ponerse en ebriedad. Nuevamente la discusión gira en torno a la responsabilidad por la acción misma, independientemente del resultado. el resultado esperado. No me parece coherente que la producción de un resultado - aun cuando pretendido- por sí sola genere responsabilidad moral. Si se cree en la libertad y se defiende una forma de indeterminismo inminente en la relación entre la elección y la acción ${ }^{30}$, no puede ser que el juicio de responsabilidad recaiga sobre lo que no se tiene control, pues aquello que es incontrolable difícilmente puede hacer parte del ámbito de la libertad. Si se descarta un determinismo basado en leyes naturales que rigen las consecuencias de nuestros actos en el mundo natural, y se constata que lo verdaderamente relevante en el juicio de responsabilidad moral tiene que ver con el ámbito psicológico del ser humano, no parece sensato desprender del solo acaecimiento de un cambio en el "mundo exterior" un mayor grado de reproche.

Teniendo en cuenta que aún en casos en los cuales hay éxito en las pretensiones, los resultados siguen estando fuera del control del agente -como en el ejemplo de Benito-, no parece adecuado, ni mucho menos útil, seguir enfocando el estudio de la responsabilidad moral -y en general la pregunta por el determinismo- en la producción de resultados. Ejemplos como el de la paloma, arriba esbozado, permiten constatar que la producción de resultados diferentes ante la misma acción, bajo las mismas circunstancias, no puede sino deberse a la suerte, y difícilmente podría aceptarse que la responsabilidad moral es una lotería. Más aún, incluso en los falsos casos de responsabilidad por producción de un resultado involuntario, similares al ejemplo propuesto, se puede constatar que lo relevante

Bien sea al estilo de Kane o no. 
es siempre la acción del agente sin respecto al resultado.

Aunque pueda ser cierto que "no es lo mismo intentar matar que matar", esto solo sería justificación para un mayor reproche si se constata que el actuar del agente varió de alguna u otra forma con respecto a aquel que hubiera producido el resultado bajo las mismas circunstancias. En tal caso, el reproche seguiría siendo el actuar del agente, y no la producción o no del resultado. Creo que por todo lo planteado en este trabajo, puede constatarse que aún habiendo o no resultados físicos determinados en el mundo exterior, la relevancia de la responsabilidad moral radica en el plano psicológico. No puede mantenerse de forma válida la responsabilidad moral en un mundo determinado físicamente en el cual el énfasis está dado en el resultado. No tiene sentido la responsabilidad sin libertad, como tampoco la libertad sin control.

Hablar de "resultados involuntarios" no aporta mucho al debate en cuestión, como ya se ha constatado. Esto implica que la relación entre voluntad y resultado es, en realidad, una falacia en la cual se hace extender la voluntad a eventos que se encuentran por fuera de su alcance. Lo cierto es que, en todos los casos, la voluntad (y con ello la libertad) se predica únicamente de la acción. En consecuencia, es la relación entre la voluntad y la acción la única que debe mediar a la hora de abordar el determinismo de cara a la responsabilidad moral.

Sea cual fuere la postura adoptada dentro del debate general entre determinismo e indeterminismo, en aras de ofrecer una solución con argumentos sólidos es menester apoyarse en dos criterios: el primero, el enfoque psicológico como forma de dar cuenta de la dualidad en la conducta del ser humano y, segundo, el enfoque basado en la acción como forma de dar cuenta del sentido del reproche en el juicio de responsabilidad $^{31}$.

\section{Bibliografía}

Blanshard, Brand. The Case of Determinism. En: Determinism and Freedom in the Age of Modern Science. Hook, S. (ed.). Nueva York: Collier-Macmillan, 1958.

EARMAN, John. A Primer on Determinism. Capítulo II "Defining Determinism". Dordrecht: Reidel Publishing Company, 1986.

Ferrante, Marcelo. Recasting the Problem of Resultant Luck. En: Legal Theory. Vol. 15, 2009, pág. 267.

FrankfuRT, Harry. La importancia de lo que nos preocupa. En: La importancia de lo que nos preocupa. Trad. Verónica Inés Weinstabl y Servanda María de Hagen. Buenos Aires: Katz Editores, 2006.

31 Al finalizar cabe reiterar lo que se ha venido estableciendo desde la introducción de este estudio: lo dicho vale para la responsabilidad moral y solo esta, pues consideraciones extramorales propias de otros ámbitos de responsabilidad impedirian exportar libremente las conclusiones a las cuales ahora se arriba. Las reglas que rigen lo moral no pueden exportarse a otros espacios sin antes hacer ajustes que parten de las necesidades y fines propios a cada uno de ellos. Por esto, sería un craso error aplicar sin reparo las conclusiones aquí esbozadas a ámbitos como el jurídico, político, económico o religioso. En este ensayo se exponen consideraciones propias de la filosofía moral y teoría jurídica que esperan enriquecer la discusión en otras esferas, bajo el entendido de que cada debate debe discurrir a su propio ritmo y con sus propias consideraciones, que no siempre han de coincidir con las aquí propuestas. 
Heisenberg, Werner. Physics and Philosophy. Capítulo II "The History of Quantum Theory". Nueva York: Harper and Brothers Publishers, 1958.

__. Physics and Philosophy. Capítulo III "The Copenhagen Interpretation of Quantum Theory". Nueva York: Harper and Brothers Publishers, 1958.

HegeL, Georg W. Líneas fundamentales de la fiIosofía del Derecho. Trad. F. E. G. Vicent. Madrid: Revista de Occidente, 1935.

KAnE, Robert. "Responsibility, Luck and Chance". En: Journal of Philosophy. Vol. 96. No. 5., 1999.

LEWIS, David K. Humean Supervenience debugged. En: LewIS, David. Papers in Metaphysics and Epistemology. Nueva York: Cambridge UP, 1999.

MorA, Carlos J. Alternativas, responsabilidad y respuesta a razones. En: Ideas y valores.
Universidad Nacional de Colombia. Vol. 58, no. 141, 2009. Disponible en: http://www.revistas.unal.edu.co/index.php/idval/article/ viewFile/12768/13370. Consultado por última vez el día 21 de marzo de 2012.

Nagel, Thomas. Moral Luck. En: Nagel, Thomas. Mortal Questions. Nueva York: Cambridge UP, 1993.

Popper, Karl. Conocimiento: subjetivo contra objetivo. En: Miller, David (comp.). POPPER: escritos selectos. México: Fondo de Cultura Económica, 1995.

PoPper, Karl. The Open Universe. Capítulo I “Kinds of Determinism". Londres: Routledge, 1991.

Sancinetti, Marcelo. Responsabilidad por acciones o responsabilidad por resultados. Bogotá: Universidad Externado de Colombia, 1996. 